\title{
Comparison of Stimulation Patterns for FES-cycling using Measures of Oxygen Cost and Stimulation Cost
}

\author{
K. J. Hunt ${ }^{1,2}$, C. Ferrario ${ }^{1}$, S. Grant ${ }^{3}$, B. Stone ${ }^{1}$, A. N. McLean ${ }^{2}$, M. H. Fraser ${ }^{2}$, \\ D. B. Allan ${ }^{2}$
}

${ }^{1}$ Centre for Rehabilitation Engineering

Department of Mechanical Engineering

University of Glasgow

Glasgow G12 8QQ

k.hunt@mech.gla.ac.uk

${ }^{2}$ Queen Elizabeth National Spinal Injuries Unit

Southern General Hospital

1345 Govan Road

Glasgow G51 4TF

${ }^{3}$ Centre for Exercise Science and Medicine

Faculty of Biomedical and Life Sciences

University of Glasgow

Glasgow G12 8QQ

\begin{abstract}
Aim: The energy efficiency of FES-cycling in spinal cord injured subjects is very much lower than that of normal cycling, and efficiency is dependent upon the parameters of muscle stimulation. We investigated measures which can be used to evaluate the effect on cycling performance of changes in stimulation parameters, and which might therefore be used to optimise them. We aimed to determine whether oxygen-cost and stimulation-cost measurements are sensitive enough to allow discrimination between the efficacy of different activation ranges for stimulation of each muscle group during constant-power cycling.
\end{abstract}

Methods: We employed a custom FES-cycling ergometer system, with accurate control of cadence and stimulated exercise workrate. Two sets of muscle activation angles ("stimulation patterns"), denoted "P1" and "P2", were applied repeatedly (eight times each) during constant-power cycling, in a repeated measures design with a single paraplegic subject. Pulmonary oxygen uptake was measured in real time and used to determine the oxygen cost of the exercise. A new measure of stimulation cost of the exercise is proposed, which represents the total rate of stimulation charge applied to the stimulated muscle groups during cycling. A number of energy-efficiency measures were also estimated.

Results: Average oxygen cost and stimulation cost of P1 were found to be significantly lower than those for $\mathrm{P} 2$ (paired t-test, $\mathrm{p}<0.05$ ): oxygen costs were $0.56 \pm 0.03 \mathrm{l} \cdot \mathrm{min}^{-1}$ and $0.61 \pm 0.04 \mathrm{l} \cdot \mathrm{min}^{-1}$ (mean $\left.\pm \mathrm{sd}\right)$, respectively; stimulation costs were $74.91 \pm 12.15$ $\mathrm{mC} \cdot \mathrm{min}^{-1}$ and $100.30 \pm 14.78 \mathrm{mC} \cdot \mathrm{min}^{-1}$ (mean $\pm \mathrm{sd}$ ), respectively. Correspondingly, all efficiency estimates for P1 were greater than those for P2.

Conclusion: Oxygen- and stimulation-cost measures both allow discrimination between the efficacy of different muscle activation patterns during constant-power FES-cycling. However, stimulation cost is more easily determined in real time, and responds more rapidly and with greatly improved signal-to-noise properties than the ventilatory oxygen uptake measurements required for estimation of oxygen cost. These measures may find utility in the adjustment of stimulation patterns for achievement of optimal cycling performance. 


\section{Introduction}

In the first half of the 1980s it was established that people with a spinal cord injury (SCI) are able to produce cyclical leg motion by means of controlled sequential stimulation of the large leg-actuating muscles; typically, the quadriceps, hamstrings and gluteal muscle groups are activated. We refer to this as FES-cycling, where FES denotes functional electrical stimulation. A number of subsequent investigations have studied the physiological adaptations which can occur in response to regular cycling exercise. Significant improvements in cardiopulmonary status (as indicated by increased peak oxygen uptake [1] and faster oxygen uptake response kinetics [2]) and in tibial bone density [3] have been observed. A recent review [4] highlights a number of additional therapeutic benefits.

While most previous studies have utilised stationary FES-cycling ergometers, a number of mobile devices have also been proposed $[5,6,7,8,9]$, raising the possibility that FES-cycling might become a recreational activity.

It has previously been noted that the efficiency of FES-cycling, i.e. the ratio of external work output to metabolic energy input, is very much lower than that of able-bodied subjects cycling under volitional control. Glaser et al. [10] found that the net efficiency ${ }^{1}$ of SCI subjects in FES-cycling was approximately $5 \%$, while able-bodied subjects performing volitional cycling on the same ergometer at similar workrates had net efficiency values of up to $20 \%$. These results are corroborated in a study by Raymond et al. [11]; a group of SCI subjects achieved an average power output of $9.2 \mathrm{~W}$ during FES-cycling with an oxygen cost of $0.75 \mathrm{l} \cdot \mathrm{min}^{-1}$. A group of able-bodied subjects cycling volitionally on the same ergometer at a similar oxygen cost achieved $42.8 \mathrm{~W}$. This corresponds to net efficiency values of approximately $5 \%$ and $22 \%$, respectively.

Care must be exercised in the interpretation of the approximate efficiency values quoted above. Theisen et al. [12], for example, have shown that efficiency can vary considerably during prolonged, constant-stimulation FES-cycling exercise. In a study with 5 SCI subjects they demonstrated that the net efficiency progressively increased up to a maximum of almost $9 \%$ after $40 \mathrm{~min}$. of cycling. Moreover, Glaser et al. [10] caution that these figures may overestimate the efficiency of cycling, since the calculations are based upon an assumption of steady-state aerobic metabolism. In the SCI population, however, blood-lactate levels are known to be elevated early in FES-cycling exercise [13], indicating an energy contribution from anaerobic metabolism which is neglected in the efficiency calculations. Nevertheless, it remains clear that FES-cycling is very inefficient.

Glaser et al. [10] have argued that the low efficiency of FES-cycling is of little consequence in the context of stationary ergometry, and may in fact be advantageous: despite the low external workrate of SCI subjects in comparison to able-bodied subjects, high metabolic and cardiopulmonary responses can be elicited. Clearly, however, maximisation of cycling efficiency is of the utmost importance in mobile cycling, where effective production of external work is required for propulsion of the rider-cycle mass.

Several factors contribute to the poor efficiency of FES-cycling. Following paralysis, muscles can become weak as a result of disuse atrophy. In addition, histochemical changes occur which result in a shift towards fast-twitch, readily-fatiguable muscle fibres [14]. The effect of these changes is exacerbated by the inverse recruitment order which occurs in FES-activated muscle fibres. Paralysed muscle generally displays some degree of spasticity; inappropriate muscle contraction can therefore be induced by the cycling motion, or by the externally-applied stimulation. FES-cycling usually involves the activation of a small number of muscle groups,

\footnotetext{
${ }^{1}$ A precise definition of net efficiency, and of other efficiency measures, can be found in section 2.6.
} 
some of which display co-activation of joints, with unwanted antagonistic effects. This contrasts with volitional cycling in which a large number of individual muscles are activated, and muscle synergy at joints is carefully coordinated.

Kjaer et al. [15] investigated the performance of eight healthy young males in both volitional and FES-induced cycling. The group performed volitional cycling at a workrate which elicited an average oxygen uptake of $1.9 \mathrm{l} \cdot \mathrm{min}^{-1}$. Complete epidural anaesthesia was then administered, resulting in total leg paralysis. Cycling was subsequently achieved by means of FES at a workrate which elicited the same oxygen uptake rate of $1.9 \mathrm{l} \cdot \mathrm{min}^{-1}$. The average workrate for this oxygen cost was found to be approximately $120 \mathrm{~W}$ during volitional cycling, but less than $40 \mathrm{~W}$ for FES-cycling. From data presented in the paper, [15], we estimate the net efficiencies for FES-cycling and for volitional cycling in this group to be $7 \%$ and $22 \%$, respectively. This establishes that even in subjects where the underlying muscle condition is normal, FES-induced cycling is less efficient by a factor of approximately 3 than cycling achieved by volitional muscle control. These results suggest that the low efficiency of FES-cycling is primarily due to the recruitment properties of electrically-stimulated muscle, together with the unfavourable biomechanics and limited muscle coordination of the FES-cycling arrangement, and, related to this, the choice of several adjustable stimulation parameters as discussed below.

There are several adjustable parameters associated with the control and coordination of muscle stimulation during cycling, and each of these will have some effect on the resulting efficiency. The stimulation parameters include: the stimulation frequency, or, if the frequency is not constant, the variable inter-pulse interval; the choice of pulsewidth and/or pulse-amplitude modulation; and the timing of muscle-group stimulation, i.e. the choice of crank-angle ranges over which each muscle group is activated. Eser et al. [16] have previously investigated the effect of stimulation frequency on power output and endurance and found that the mean power output during $30 \mathrm{~min}$. of FES-cycling was significantly greater at $50 \mathrm{~Hz}$ and $60 \mathrm{~Hz}$ than at $30 \mathrm{~Hz}$.

There is considerable scope for the development of measures and methods which can be used to evaluate the effect on cycling performance of changes in these parameters, and which might therefore be used to optimise them. Thus, in the present work we investigated outcome measures which might be used to characterise the efficacy of a given set of parameters, and which might be sensitive enough to reflect changes in the parameters.

The present work focused on the selection of muscle activation angles only. Previous studies have analysed electromyographic (EMG) data from normal subjects during volitional cycling to identify muscle timing characteristics $[17,18,19,6,20]$, and have attempted to use the results to design activation ranges for SCI subjects performing FES-cycling on the same ergometer device. Two of these studies, [6, 20], found that the activation patterns obtained during normal, volitional cycling had to be manually adjusted in order to achieve smooth cycling motion with paraplegic subjects. Petrofsky's studies $[17,18,19]$ each reported measurement of EMG activation patterns from four normal subjects, and the apparently successful use of these to set the timing and stimulation intensity variables for paraplegic cycling. Surprisingly, the maximum power outputs achieved were very much higher than those reported elsewhere (e.g. approximately $100 \mathrm{~W}$ power output is reported in [19]).

Other groups have attempted to numerically optimize activation angles by maximizing an analytical cost function which captures mechanical output forces [21, 22, 23, 24]. This approach is based upon a dynamic simulation model of lower-limb cycling, and has not thus far been experimentally verified. Alternatively, Gföhler et al. [25] have empirically measured output forces in response to stimulation over large angle ranges, and have used these results to choose desirable activation ranges.

In the present work we empirically studied the selection of muscle activation angles using 
the following variables: real-time, breath-by-breath measurement of pulmonary oxygen uptake rate (denoted $\dot{\mathrm{VO}}_{2}$ ), which gives the oxygen cost of the exercise; a new real-time measure of total stimulation charge rate, which can be viewed as the stimulation cost; and estimates of cycling efficiency. In particular, the aim of this work was to determine whether oxygen-cost and stimulation-cost measurements are sensitive enough to allow discrimination between the efficacy of different activation ranges for stimulation of each muscle group during constant-power cycling.

\section{Methods}

\subsection{Apparatus}

We utilized a recumbent tricycle (Inspired Cycle Engineering Ltd., UK), adapted for paraplegic FES-cycling and arranged for static ergometry [26] (see figure 1). The tricycle is equipped

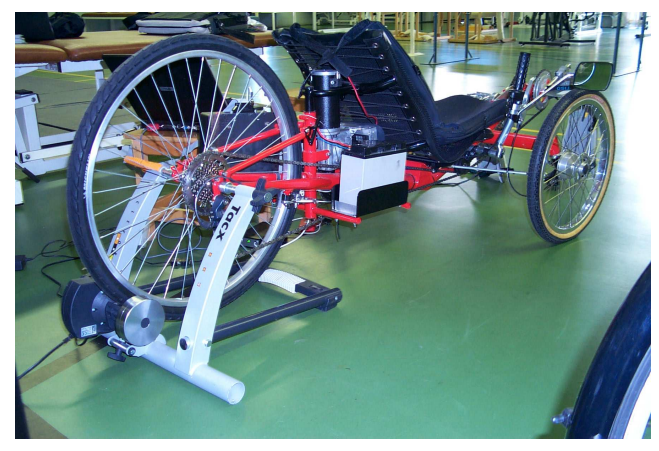

(a) Physical apparatus.

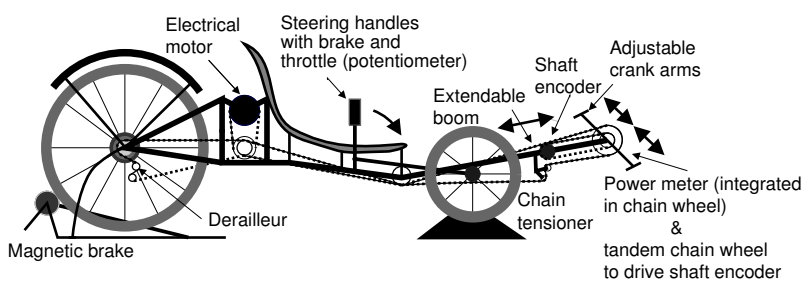

(b) Schematic diagram.

Figure 1: Motorised and instrumented recumbent tricycle for exercise testing during FEScycling.

with an electric motor, connected through gearing to a rear drive wheel, and coupled to the cranks at the front of the tricycle. Thus, energy input to the system from the motor and from the stimulated leg muscles combine to work against an electronically-controlled load (Tacx, Holland) at the drive wheel. A shaft encoder provides continuous measurement of crank angle and cadence, while a sensor integrated in the crank measures the power output resulting from the combined effects of leg stimulation and the inertia of the legs. The system is interfaced to a PC which provides real-time feedback control of cycling cadence (by continuously altering the motor command signal) and of leg power (via automatic adjustment of stimulation intensity), according to the control structure shown in figure 2 .

With this arrangement, testing can be carried out under conditions of precisely controlled cadence and leg-power output. Full technical details of these feedback control systems and a discussion of the utility of the system for exercise testing are given in [26].

\subsection{Subjects}

The study design is repeated measures in one paraplegic subject. The subject had a clinicallycomplete traumatic spinal cord lesion at neurological level T10. He was 59 years old and 5 years post-injury at the time of the study. He had been participating in an FES-cycling 


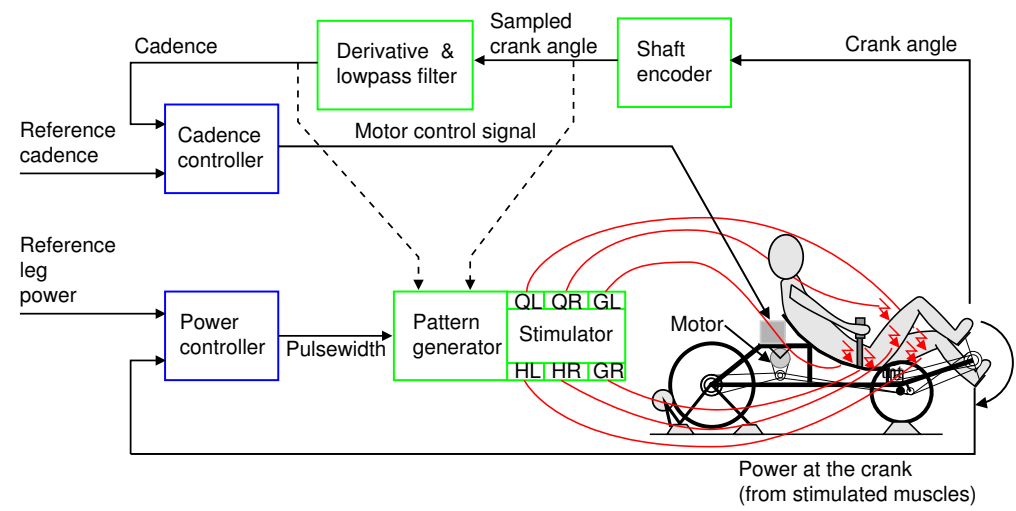

Figure 2: Integrated closed-loop control scheme. One loop automatically adjusts the motor input to keep the cycling cadence close to a setpoint value. The second loop automatically adjusts the stimulation pulsewidth to keep the leg power close to an arbitrary reference value. Stimulated muscle group are indicated as follows: QL - Quadriceps Left; QR - Quadriceps Right; HL - Hamstrings Left; HR - Hamstrings Right; GL - Gluteus Left; GR - Gluteus Right.

programme, cycling once per week for up to one hour, for more than two years prior to the experiments reported here. The programme was approved by the Southern General Hospital ethics committee, and the subject provided informed consent prior to participation.

\subsection{Stimulation parameters}

Pairs of surface electrodes (round, $7 \mathrm{~cm}$ diameter) were attached to each of six muscle groups, i.e. the left and right quadriceps, hamstring and gluteal muscles. Electrodes were positioned in relation to anatomical markers (e.g. top of patella for quadriceps electrodes), and with a fixed distance between each electrode pair. To achieve an effective and smooth cycling motion the muscle groups must be switched on and off at appropriate times during the 360 deg crank cycle, using the continuous crank-angle measurement. In this work we evaluated two stimulation patterns, denoted "P1" and "P2", at a constant cadence of $50 \mathrm{rpm}$. The patterns are shown schematically in figure 3. The start and stop angles with P1 and P2 for the muscle groups in the right leg are given in table 1 . Thus, the angle ranges during which the quadriceps and

\begin{tabular}{|c|c|c|c|}
\hline \hline Pattern & Quadriceps & Hamstring & Gluteus \\
\hline \hline P1 & $10^{\circ}-110^{\circ}$ & $123^{\circ}-200^{\circ}$ & $45^{\circ}-135^{\circ}$ \\
\hline P2 & $355^{\circ}-125^{\circ}$ & $108^{\circ}-215^{\circ}$ & $45^{\circ}-135^{\circ}$ \\
\hline \hline
\end{tabular}

Table 1: Stimulation patterns for the three groups of muscles (right side).

hamstring muscles were activated in pattern P2 were each extended by 30 degrees with respect to pattern P1. Pattern P1 had previously been determined by manual adjustment to provide smooth and effective cycling with the subject studied here, and P2 represents an arbitrary perturbation from this nominal pattern.

We utilised a constant stimulation frequency of $20 \mathrm{~Hz}$, with monophasic charge-balanced pulses, and the following current amplitudes for each channel: Q - $120 \mathrm{~mA}, \mathrm{H}-110 \mathrm{~mA}$, and G - $90 \mathrm{~mA}$. The stimulation pulsewidth was varied in real time by feedback control (see figure 2) 


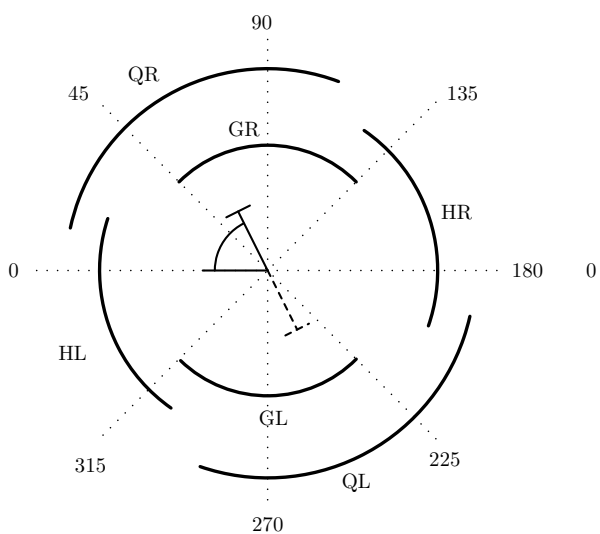

(a) Stimulation pattern P1.

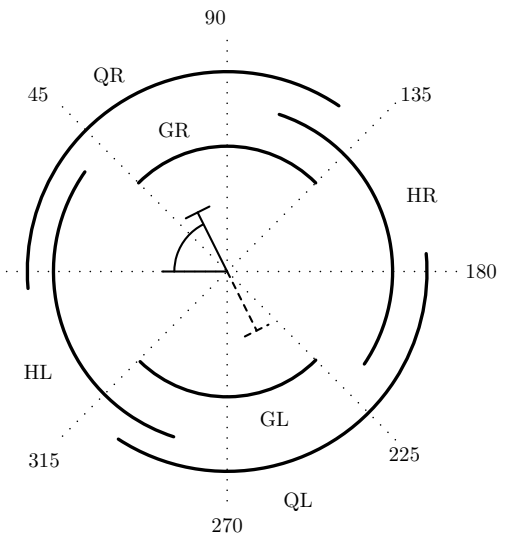

(b) Stimulation pattern P2.

Figure 3: Stimulation patterns for $50 \mathrm{rpm}$ : QL -Quadriceps Left; QR - Quadriceps Right; HL - Hamstrings Left; HR - Hamstrings Right; GL - Gluteus Left; GR - Gluteus Right.

within the range 0-700 $\mu$ s in order to achieve a pre-specified exercise workrate (i.e. leg power output) and was the same for all muscles.

\subsection{Evaluation protocol}

The test protocol for evaluation of patterns P1 and P2 was preceded by a stimulated warm-up of 6 minutes cycling at a constant low-level workrate (approximately $+5 \mathrm{~W}$ ), while the motor and its control loop maintained a cadence of $50 \mathrm{rpm}$. Warm-up was followed by a 9-minute rest period, and then a further $6 \mathrm{~min}$. of recorded rest (i.e. the subject was connected to the cardiorespiratory monitoring system). Starting with the recorded rest period, the test followed the protocol illustrated in figure 4 .

Following rest, there was an initial passive cycling phase for $4 \mathrm{~min}$. in order to accommodate the leg muscles and their possible spastic reflexes to the cyclic motion, with only the motor driving the legs and no stimulation applied, and then a stimulated cycling phase for 6 min. at a workrate of $0 \mathrm{~W}$. It should be noted that the measured power output at the cranks during passive, non-stimulated cycling is negative (in these tests, it was approximately $-6 \mathrm{~W}$ ), representing the rate of energy input required to move the mass of the legs. Thus, at a workrate of $0 \mathrm{~W}$ a certain, non-zero, level of stimulation is required.

After the $0 \mathrm{~W}$ phase the subject cycled at a constant workrate of $8 \mathrm{~W}$, which corresponds to a total effective workrate of $14 \mathrm{~W}$ with respect to passive, for $24 \mathrm{~min}$. (the "evaluation phase"). During each cycling phase (i.e. from 6 min. onwards) the motor and its controller maintained a constant cadence of $50 \mathrm{rpm}$, independent of the level of stimulation. During the evaluation phase each stimulation pattern was applied for $6 \mathrm{~min}$., in order to allow the gas exchange responses to reach a steady state, and then an instantaneous switch was made to the other pattern. During each 24-min. evaluation phase, the patterns were applied either in the order P1-P2-P1-P2 or P2-P1-P2-P1, as indicated in figure 4.

A total of four tests were carried out, each on a separate day. Two of the tests consisted of the sequence P1-P2-P1-P2 and in the other two tests the patterns were applied in the order $\mathrm{P} 2-\mathrm{P} 1-\mathrm{P} 2-\mathrm{P} 1$, resulting in the availability of eight 6 -min. stages with $\mathrm{P} 1$ and seven 6 -min. stages with P2 (one P2 stage was not completed). 


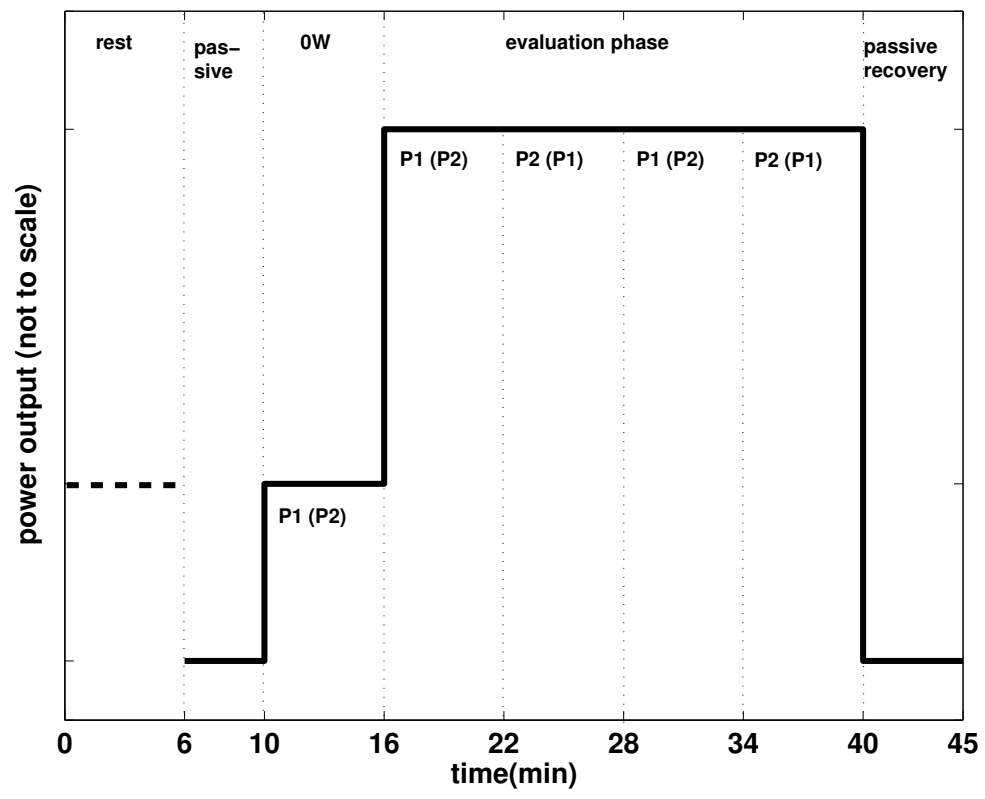

Figure 4: Schematic diagram an individual pattern evaluation test protocol with patterns applied in the order P1-P2-P1-P2 (or P2-P1-P2-P1, as indicated in brackets).

\subsection{Gas Exchange measurements}

Using a portable breath-by-breath system (MetaMax 3B, Cortex Biophysik GmbH, Germany) and with the subject breathing through a low dead-space mask, respired $\mathrm{O}_{2}$ and $\mathrm{CO}_{2}$ concentrations and respired volume and flow were measured continuously using discrete gas analyzers and a turbine, respectively. Pulmonary gas exchange variables were derived breath-by-breath on a real time basis.

\subsection{Data analysis and outcome measures}

The oxygen uptake responses for each test were averaged over the final minute of each 6-min. stage in the evaluation phase. We refer to this variable as the oxygen cost of the work.

The total electrical charge delivery rate for the stimulated muscle groups during exercise was calculated as follows. The charge for each individual pulse is obtained from the product of instantaneous pulsewidth and current. These values were summed over the six muscle groups, and then due account taken of the on/off times of each group during each crank rotation. The stimulation cost of the exercise is defined as the average value of this total charge rate over a given time interval. Here, the stimulation cost was taken as the average over the final minute of each stage in the evaluation phase.

The efficiency of exercise, defined generally as the ratio of work accomplished to energy expended within a given time interval under conditions of steady-state aerobic exercise, is defined here as follows:

$$
\begin{gathered}
\text { Total Gross Efficiency: } \epsilon_{g}^{t}=\frac{P^{t}}{P^{e}} \times 100 \% \\
\text { Total Net Efficiency: } \epsilon_{n}^{t}=\frac{P^{t}}{P^{e}-P^{p}} \times 100 \%
\end{gathered}
$$




$$
\begin{gathered}
\text { Gross Efficiency: } \epsilon_{g}=\frac{P^{+}}{P^{e}} \times 100 \% \\
\text { Net Efficiency: } \quad \epsilon_{n}=\frac{P^{+}}{P^{e}-P^{r}} \times 100 \%
\end{gathered}
$$

Here, $P^{t}$ is the total workrate range (measured from the negative passive power), while $P^{+}$is the absolute (positive) value measured during the stimulated evaluation phase. $P^{e}$ is the rate of aerobic metabolic energy expenditure of the subject during exercise in the evaluation phase and the rate of metabolic energy expenditure during passive, non-stimulated cycling is $P^{p} . P^{r}$ represents the rate of aerobic metabolic energy expenditure of the subject at rest. Note that the net efficiency values quoted in section 1 are based upon equation (4).

The rates of metabolic energy expenditure in the above equations $\left(P^{e}, P^{p}\right.$ and $\left.P^{r}\right)$ were estimated here using steady-state oxygen uptake and carbon dioxide output in each condition, with values averaged over the final minute of each evaluation phase.

A paired-t test was used to compare the outcome measures for the two different patterns to determine whether any differences in sample means were statistically significant, with significance deemed to hold for $\mathrm{p}<0.05$.

\section{Results}

\subsection{Power and cadence control}

Figure 5 gives an illustration of the accuracy of feedback control of exercise workrate (power) and cadence. Data for the passive, $0 \mathrm{~W}$, and evaluation phases of Test 2 are shown (c.f. figure 4), but the power and cadence data for the other three tests are similar. The measured power and power reference signals are shown in the upper part of the figure. It can be seen that the stimulation pulsewidth (middle plot) is automatically adjusted by feedback to maintain the desired power throughout the test. The motor control loop keeps the cadence constant at $50 \mathrm{rpm}$ (bottom plot).
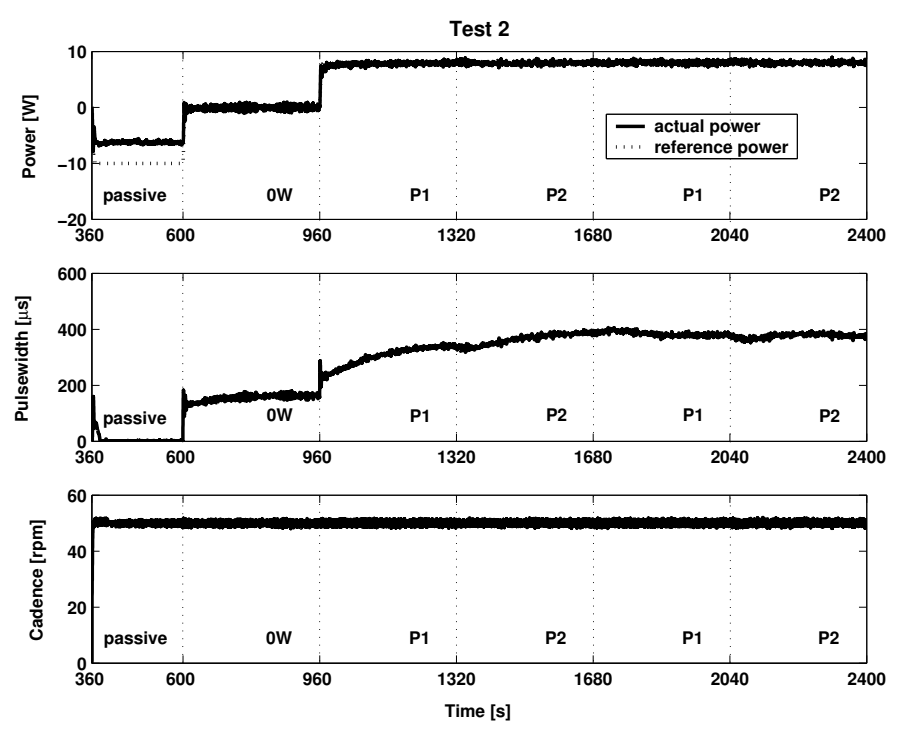

Figure 5: Time course of power and power reference, pulsewidth, and cadence during Test 2. 


\subsection{Oxygen cost}

Figure 6 shows the time-course for oxygen uptake for all four tests. The average $\mathrm{V}_{2}$ for each pattern during the final minute of each stage (the oxygen cost) of exercise is reported in table 2 .
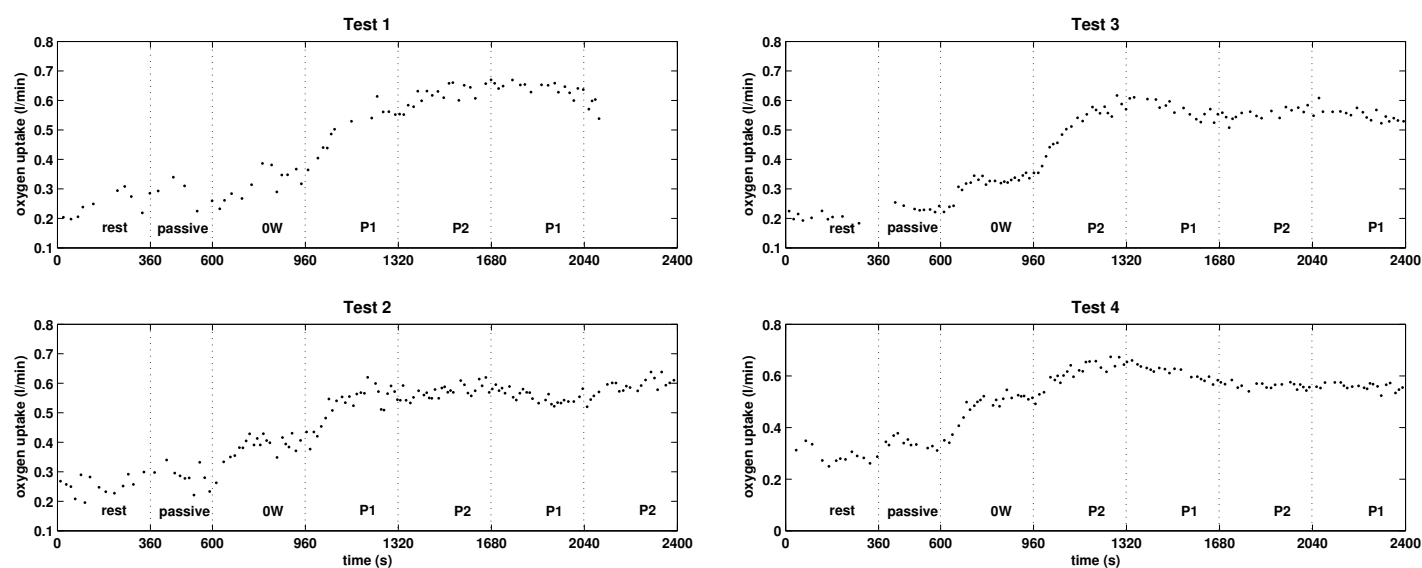

(a) Presentation order P1-P2-P1-P2

(b) Presentation order P2-P1-P2-P1

Figure 6: Time-course for the oxygen uptake during the four exercise tests.

The mean values for the eight presentations of $\mathrm{P} 1$ and seven presentations of $\mathrm{P} 2$ were $0.56 \pm 0.03$

\begin{tabular}{|c|c|c|c|c|}
\hline \hline Test nr. & 1 & 2 & 3 & 4 \\
\hline Order & $1-2-1-2$ & $1-2-1-2$ & $2-1-2-1$ & $2-1-2-1$ \\
\hline \hline Rest & 0.25 & 0.31 & 0.24 & 0.28 \\
\hline Passive & 0.26 & 0.28 & 0.23 & 0.32 \\
\hline $0 \mathrm{~W}$ & $0.35_{(\mathrm{P} 1)}$ & $0.41_{(\mathrm{P} 1)}$ & $0.34_{(\mathrm{P} 2)}$ & $0.52_{(\mathrm{P} 2)}$ \\
\hline Stage 1 & $0.56_{(\mathrm{P} 1)}$ & $0.55_{(\mathrm{P} 1)}$ & $0.59_{(\mathrm{P} 2)}$ & $0.65_{(\mathrm{P} 2)}$ \\
\hline Stage 2 & $0.66_{(\mathrm{P} 2)}$ & $0.60_{(\mathrm{P} 2)}$ & $0.55_{(\mathrm{P} 1)}$ & $0.58_{(\mathrm{P} 1)}$ \\
\hline Stage 3 & $0.63_{(\mathrm{P} 1)}$ & $0.56_{(\mathrm{P} 1)}$ & $0.57_{(\mathrm{P} 2)}$ & $0.55_{(\mathrm{P} 2)}$ \\
\hline Stage 4 & & $0.61_{(\mathrm{P} 2)}$ & $0.53_{(\mathrm{P} 1)}$ & $0.55_{(\mathrm{P} 1)}$ \\
\hline \hline
\end{tabular}

Table 2: $\dot{\mathrm{V}} \mathrm{O}_{2}$ values averaged over the last minute of each interval $\left(1 \cdot \min ^{-1}\right)$ : oxygen cost.

and $0.61 \pm 0.04\left(1 \cdot \mathrm{min}^{-1}\right.$, mean $\left.\pm \mathrm{sd}\right)$, respectively. Stage 4 for the first test is not available because the pulsewidth reached saturation during that stage.

The mean net oxygen cost values for $\mathrm{P} 1$ and $\mathrm{P} 2$, obtained by subtracting the resting level of $\dot{\mathrm{VO}}_{2}$ from the exercise level, are $0.29 \pm 0.03 \mathrm{l} \cdot \mathrm{min}^{-1}$ and $0.33 \pm 0.04 \mathrm{l} \cdot \mathrm{min}^{-1}$, respectively. The mean values of both absolute and net $\mathrm{V}_{2}$ for $\mathrm{P} 2$ were found to be significantly greater than those for $\mathrm{P} 1$, with $\mathrm{p}<0.05$.

\subsection{Stimulation cost}

Figure 7 shows the time-course for the total charge rate during all the tests, computed as described in section 2.6. The change in the value of the total charge rate delivered to the 

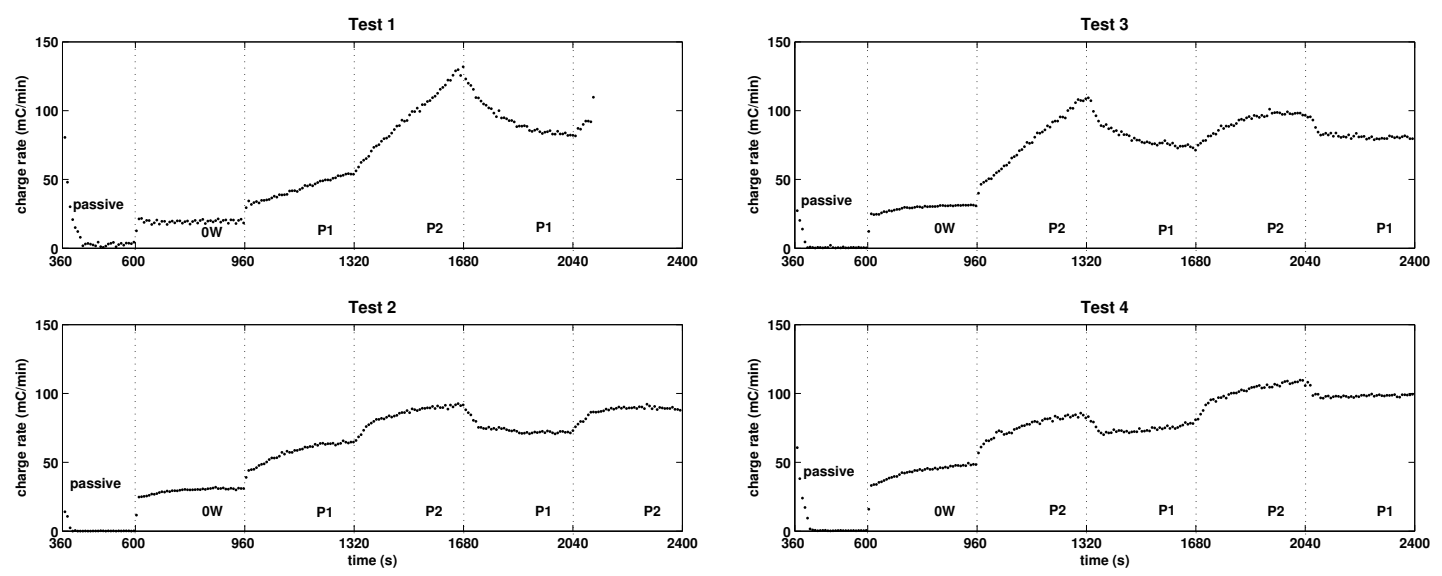

(a) Presentation order P1-P2-P1-P2

(b) Presentation order P2-P1-P2-P1

Figure 7: Time-course for the rate of total charge delivered to the muscles during the four exercise tests.

muscles depending on the pattern used is clear; the charge rate increases when P2 is used and decreases when $\mathrm{P} 1$ is used.

\begin{tabular}{|c|c|c|c|c|}
\hline \hline Test nr. & 1 & 2 & 3 & 4 \\
\hline Order & $1-2-1-2$ & $1-2-1-2$ & $2-1-2-1$ & $2-1-2-1$ \\
\hline \hline 0W & $19.58_{(\mathrm{P} 1)}$ & $30.88_{(\mathrm{P} 1)}$ & $31.21_{(\mathrm{P} 2)}$ & $48.25_{(\mathrm{P} 2)}$ \\
\hline Stage 1 & $53.59_{(\mathrm{P} 1)}$ & $64.68_{(\mathrm{P} 1)}$ & $106.20_{(\mathrm{P} 2)}$ & $83.42_{(\mathrm{P} 2)}$ \\
\hline Stage 2 & $127.13_{(\mathrm{P} 2)}$ & $91.08_{(\mathrm{P} 2)}$ & $73.44_{(\mathrm{P} 1)}$ & $77.52_{(\mathrm{P} 1)}$ \\
\hline Stage 3 & $83.39_{(\mathrm{P} 1)}$ & $72.30_{(\mathrm{P} 1)}$ & $97.84_{(\mathrm{P} 2)}$ & $107.51_{(\mathrm{P} 2)}$ \\
\hline Stage 4 & & $88.93_{(\mathrm{P} 2)}$ & $80.73_{(\mathrm{P} 1)}$ & $93.67_{(\mathrm{P} 1)}$ \\
\hline \hline
\end{tabular}

Table 3: Total charge rate for all muscles averaged over the last minute of each stage, measured in $\mathrm{mC} \cdot \mathrm{min}^{-1}$ : stimulation cost.

The rate of delivery of total charge, averaged over the last minute of each stage (the stimulation cost), is reported in table 3. Mean values for the rate of charge delivered when using patterns $\mathrm{P} 1$ and $\mathrm{P} 2$ are $74.91 \pm 12.15$ and $100.30 \pm 14.78\left(\mathrm{mC} \cdot \mathrm{min}^{-1}\right.$, mean $\left.\pm \mathrm{sd}\right)$, respectively. The mean values for P2 were found to be significantly greater than those for P1, with $\mathrm{p}<0.05$.

Figure 8 shows the time course of the pulsewidth for all the tests. It can be seen that in most of the cases when the pattern was switched from P1 to P2 the pulsewidth immediately decreased, only to rise again after about one minute. The converse is the case when changing from pattern $\mathrm{P} 2$ to $\mathrm{P} 1$. This is a reflection of the longer stimulation on-time (P2) requiring a lower instantaneous pulsewidth after a change from $\mathrm{P} 1$, while maintaining a constant power, before the confounding effects of differing efficiency and fatigue properties of the two patterns intervene.

The values for average pulsewidth during the last minute of each stage are reported in table 4. Mean values for the pulsewidth when using pattern P1 and P2 are 392.56 \pm 63.07 and $423.30 \pm 62.26$ ( $\mu \mathrm{s}$, mean $\pm \mathrm{sd}$ ), respectively. Thus, the pulsewidth for pattern P1 is lower than 

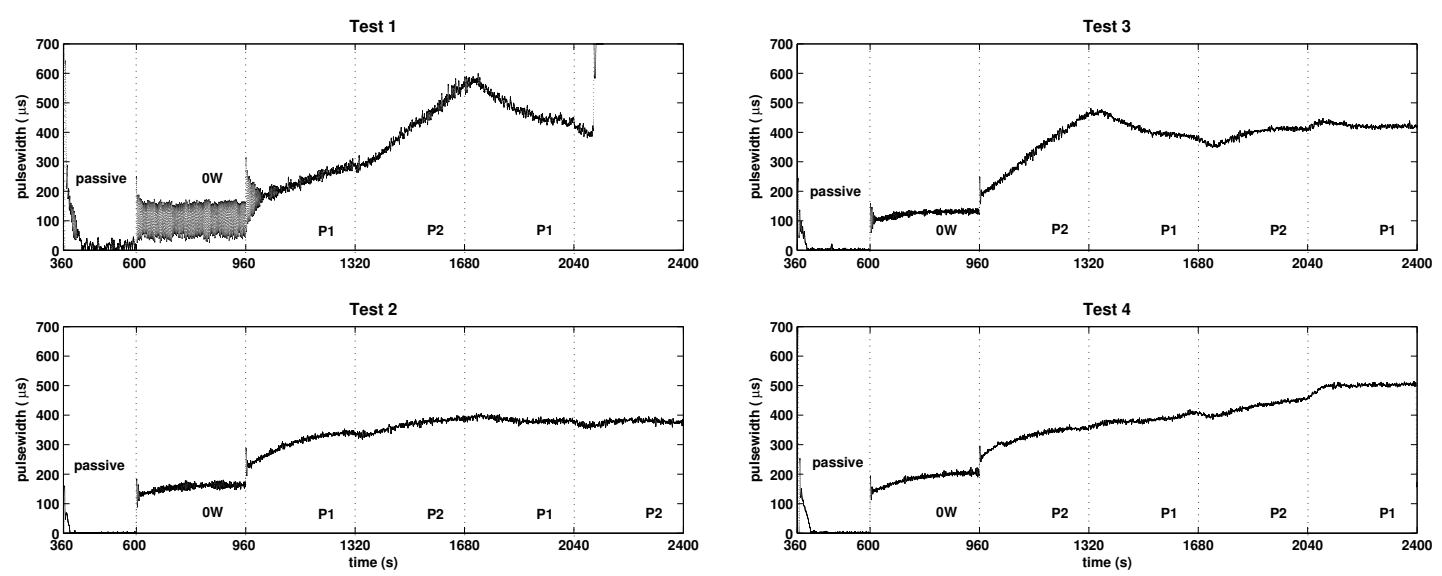

(a) Presentation order P1-P2-P1-P2

(b) Presentation order P2-P1-P2-P1

Figure 8: Time-course for the pulsewidth during the four exercise tests.

\begin{tabular}{|c|c|c|c|c|}
\hline \hline Test nr. & 1 & 2 & 3 & 4 \\
\hline Order & $1-2-1-2$ & $1-2-1-2$ & $2-1-2-1$ & $2-1-2-1$ \\
\hline \hline 0W & $104.98_{(\mathrm{P} 1)}$ & $162.13_{(\mathrm{P} 1)}$ & $132.24_{(\mathrm{P} 2)}$ & $204.89_{(\mathrm{P} 2)}$ \\
\hline Stage 1 & $281.43_{(\mathrm{P} 1)}$ & $339.25_{(\mathrm{P} 1)}$ & $443.67_{(\mathrm{P} 2)}$ & $354.20_{(\mathrm{P} 2)}$ \\
\hline Stage 2 & $539.71_{(\mathrm{P} 2)}$ & $386.63_{(\mathrm{P} 2)}$ & $385.66_{(\mathrm{P} 1)}$ & $404.49_{(\mathrm{P} 1)}$ \\
\hline Stage 3 & $439.41_{(\mathrm{P} 1)}$ & $380.51_{(\mathrm{P} 1)}$ & $411.83_{(\mathrm{P} 2)}$ & $451.32_{(\mathrm{P} 2)}$ \\
\hline Stage 4 & & $375.78_{(\mathrm{P} 2)}$ & $420.45_{(\mathrm{P} 1)}$ & $489.25_{(\mathrm{P} 1)}$ \\
\hline \hline
\end{tabular}

Table 4: Average pulsewidth during the last minute of each stage, measured in $\mu \mathrm{s}$.

for pattern P2, but the difference between these values is not statistically significant.

\subsection{Efficiency}

The values for the four efficiency measures defined in equations (1)-(4) are reported in figure 9 . All four measures indicate that P1 has a higher efficiency than P2, but only the differences for the two gross efficiency measures were determined to be significant $(\mathrm{p}<0.05)$.

\section{Discussion}

Our observations suggest that both oxygen cost and stimulation cost of constant-power FES cycling can be appreciably influenced by the muscle stimulation paradigm employed and that these cost measurements may provide sufficient discriminatory power to allow assessment of different muscle stimulation patterns during FES-cycling exercise.

We chose to switch each pattern every 6 minutes because we wished to ensure that each stage was of sufficient duration that a true steady-state was attained; we estimated that this was the minimum time necessary for the respiratory responses to reach a steady state, based on the knowledge that SCI subjects typically have prolonged $\dot{\mathrm{V}}_{2}$ kinetics $[2,27]$. On the other 


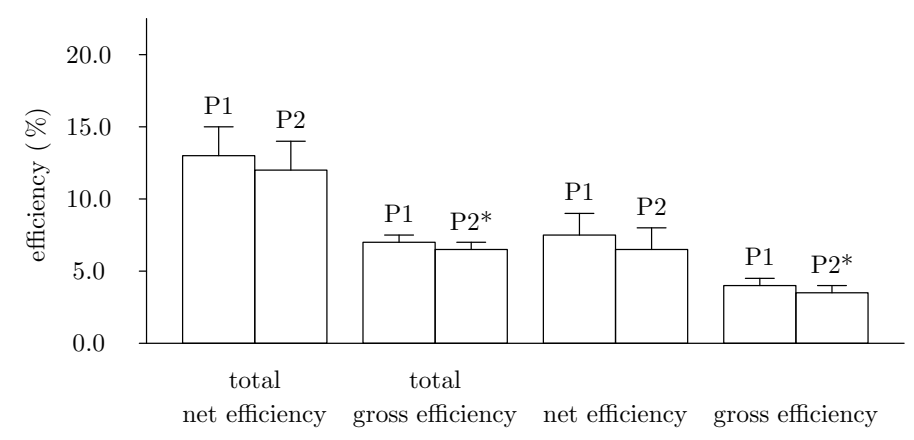

Figure 9: Bar chart showing mean efficiency measures $(+\mathrm{sd}) .{ }^{*}$ denotes a significant difference with $\mathrm{p}<0.05$.

hand, it was desirable to make the duration of each stage as short as possible to allow a greater number of pattern repetitions within a given test period. The choice of a 6-minute duration for each stage was therefore a compromise between the need to reach a steady state, and the desire to maximise the number of repetitions in each test period. The requirement for test repetition is dictated by the relatively high levels of noise which characterize breath-by-breath gas exchange measurements. Repeat tests are required to obtain response averages for improved signal-to-noise ratio and therefore improved discriminability of response differences.

The practicalities of pulmonary gas exchange measurement means that testing can be time consuming, which is an important consideration as the subject must remain connected to the specialised measurement equipment throughout each test. These considerations led us to investigate alternative indices of the cost of exercise, based on the stimulation intensity. Rather than simply analysing the stimulation pulsewidth, we developed a new measure of stimulation cost which takes account not only of pulsewidth, but also of the differing current amplitudes in each muscle group, and the on/off times of each channel during crank rotation. In the context of FES-cycling, this stimulation cost variable represents the total charge rate applied to the muscles and therefore gives a true measure of stimulation intensity.

The stimulation cost and oxygen cost variables are in agreement in identifying significant differences between the two patterns overall. Moreover, within each test sequence the cost variables were in agreement on the most (P2) and least (P1) costly patterns (c.f. tables 2 and 3). It would also appear that the stimulation cost variable responds more rapidly than oxygen cost to changes in the stimulation paradigm. It is clearly seen in several of the pattern transitions in figure 7 that the stimulation response dynamic takes approximately 1 minute. The response kinetics of $\dot{\mathrm{V}} \mathrm{O}_{2}$, while not clearly discernible in these tests due to noise (figure 6), would, as discussed above, be expected to be considerably longer.

Stimulation cost has several advantages over oxygen cost, when viewed as a means of optimising stimulation parameters to improve exercise efficacy. Stimulation cost is easy to determine in real time: it does not require the subject to be connected to any pulmonary gas exchange sensors. It appears to respond more rapidly and with greatly improved signal-to-noise characteristics, which would facilitate more accurate and rapid testing.

All four efficiency measures show that P1 is more efficient than P2, but the differences were found to be significant only for the two gross efficiency measures. This is perhaps surprising, since the efficiency measures are essentially scaled versions of the oxygen cost of the work, suggesting that this scaling itself reduces the effective discriminatory power of oxygen uptake measures. In principle, the new measures of total efficiency introduced here, which exploit the full workrate range of the exercising muscles (starting from the negative, passive workrate), 
should give improved signal to noise characteristics for these computations and therefore a more accurate representation of the muscular and biomechanical efficacy of stimulation patterns.

Overall, the responses reported here suggest that exercise with pattern P2 is significantly less efficacious than with $\mathrm{P} 1$. This might be a result of $\mathrm{P} 2$ having much wider stimulation angles, and therefore larger areas of the crank cycle where muscle force is less effectively translated into work-producing torque (i.e. due to a reduced moment arm). In addition, P2 will result in muscle activation over a wider range of muscle lengths (including areas of activation where muscle lengths are both longer and shorter than with P1, and where force transmission may be less effective).

Analysis of the pulsewidth data also points to the poor efficiency of P2. Since with P2 each stimulation channel is switched on over a larger part of the crank cycle, one would expect that for constant-power cycling lower peak forces would require to be developed, and therefore the pulsewidth with $\mathrm{P} 2$ would be less than that for P1. The pulsewidth plots in figure 8 show that this is indeed the case in the brief periods following pattern changes: in all transitions from P1 to P2 there is a small but rapid decrease in pulsewidth. However, the combined effects of lower efficiency and more rapid fatigue then cause the pulsewidth with P2 to progressively rise, to the extent that the average pulsewidth in the final minute of each stage in the evaluation phase is actually greater with $\mathrm{P} 2$ than with $\mathrm{P} 1$.

The results may also, in part, reflect differences in muscle-type recruitment profile, but further physiological measurements would be required to resolve this issue.

\section{Conclusions}

In summary, these results raise the possibility that oxygen cost and stimulation cost measurements may allow discrimination between the efficacy of different muscle activation patterns. Moreover, it appears that the discriminatory power of the oxygen cost of exercise is reflected by a more easily determined index of cycling efficiency, i.e. the cost in terms of the applied rate of stimulation charge (and therefore the overall intensity of muscle activation). This stimulation cost variable is preferable as it is easy to determine in real time, does not require the subject to be connected to physiological measurement sensors, and appears to respond more rapidly and with greatly improved signal-to-noise properties than ventilatory oxygen uptake measurements. Further work is required with a larger group of subjects to determine if these findings are typical for the spinal cord injured population.

\section{References}

[1] T. Mohr, J. L. Andersen, F. Biering-Sorensen, H. Galbo, J. Bangsbo, A. Wagner, and M. Kjaer, "Long term adaptation to electrically induced cycle training in severe spinal cord individuals," Spinal Cord, vol. 35, pp. 1-16, 1997.

[2] T. J. Barstow, A. M. E. Scremin, D. L. Mutton, C. F. Kunkel, T. C. Cagle, and B. J. Whipp, "Changes in gas-exchange kinetics with training in patients with spinal cord injury," Med. Sci. Sports Exer., vol. 28, pp. 1221-1228, 1996.

[3] T. Mohr, J. Podenphant, F. Biering-Sorensen, H. Galbo, G. Thamsborg, and M. Kjaer, "Increased bone mineral density after prolonged electrically induced cycle training of paralyzed limbs in spinal cord injured man," Calcif. Tissue Int., vol. 61, no. 19, pp. 22-25, 1997. 
[4] T. W. J. Janssen, R. M. Glaser, and D. B. Shuster, "Clinical efficacy of electrical stimulation exercise training: Effects on health, fitness, and function," Top. Spinal Cord Injury Rehab., vol. 3, no. 3, pp. 33-49, 1998.

[5] J. S. Petrofsky, H. Heaten, and C. A. Phillips, "Outdoor bicycle for exercise in paraplegics and quadriplegics," J. Biomed. Eng., vol. 5, pp. 292-296, October 1983.

[6] D. J. Pons, C. L. Vaughan, and G. G. Jaros, "Cycling device powered by the electrically stimulated muscles of paraplegics," Med. Biol. Eng. Comput., vol. 27, pp. 1-7, January 1989 .

[7] M. Gföhler, M. Loicht, and P. Lugner, "Exercise tricycle for paraplegics," Med. Biol. Eng. Comput., vol. 36, pp. 118-121, 1998.

[8] T. A. Perkins, N. Donaldson, N. A. C. Hatcher, I. D. Swain, and D. E. Wood, "Control of leg-powered paraplegic cycling using stimulation of the lumbo-sacral anterior spinal nerve roots," IEEE Trans. Neural Sys. Rehab. Eng., vol. 10, pp. 158-164, September 2002.

[9] K. J. Hunt, T. Schauer, N.-O. Negård, W. Stewart, and M. H. Fraser, "A pilot study of lower-limb FES cycling in paraplegia," in Proc. "7th Ann. Conf. Int. Functional Electrical Stimulation Society, (Ljubljana, Slovenia), 2002.

[10] R. M. Glaser, S. F. Figoni, S. P. Hooker, M. M. Rodgers, B. N. Ezenwa, A. G. Suryaprasad, S. C. Gupta, and T. Mathews, "Efficiency of FNS leg cycle ergometry," in Proc. 11th Ann. Int. Conf. IEEE Eng. Med. Biol. Soc., pp. 961-963, 1989.

[11] J. Raymond, G. M. Davis, and M. van der Plas, "Cardiovascular responses during submaximal electrical stimulation-induced leg cycling in individuals with paraplegia," Clin. Physiol. Func. Imaging, vol. 22, no. 2, pp. 92-98, 2002.

[12] D. Theisen, C. Fornusek, J. Raymond, and G. M. Davis, "External power output changes during prolonged cycling with electrical stimulation," J. Rehab. Med., vol. 34, pp. 171-175, 2002 .

[13] R. M. Glaser, "Physiology of functional electrical stimulation-induced exercise: Basic science perspective," J. Neuro. Rehab., vol. 5, pp. 49-61, 1991.

[14] T. Martin, R. Stein, P. Hoeppner, and D. Reid, "Influence of electrical stimulation on the morphological and metabolic properties of paralyzed muscle," J. Appl. Physiol., vol. 72, pp. 1401-1406, 1992.

[15] M. Kjaer, G. Perko, N. H. Secher, R. Boushel, N. Beyer, S. Pollack, A. Horn, A. Fernandes, T. Mohr, S. F. Lewis, and H. Galbo, "Cardiovascular and ventilatory responses to electrically induced cycling with complete epidural anaesthesia in humans," Acta Physiol. Scand., vol. 151, pp. 199-207, 1994.

[16] P. Eser, N. Donaldson, H. Knecht, and E. Stüssi, "Influence of different stimulation frequencies on power output and fatigue during FES-cycling in recently injured SCI people," IEEE Trans. Neur. Sys. Rehab. Eng., vol. 11, no. 3, pp. 236-240, 2003.

[17] J. S. Petrofsky, C. A. Phillips, J. Almeyda, R. Briggs, W. Couch, and W. Colby, "Aerobic trainer with physiological monitoring for exercise in paraplegic and quadriplegic patients," J. Clin. Eng., vol. 10, no. 4, pp. 307-316, 1985. 
[18] J. S. Petrofsky and J. Smith, "Three wheel cycle ergometer for use by men and women with paralysis," Med. Biol. Eng. Comput., vol. 30, pp. 364-369, 1992.

[19] J. S. Petrofsky, "New algorithm to control a cycle ergometer using electrical stimulation," Med. Biol. Eng. Comput., vol. 41, pp. 18-27, 2003.

[20] G. C. Jang, J. J. Chen, C. T. Shih, and T. C. Huseh, "Design of FES-cycling system and its stimulation patterns," Chinese J. Med. Bio. Eng., vol. 13, pp. 305-316, December 1993.

[21] M. Gföhler and P. Lugner, "Cycling by means of functional electrical stimulation," IEEE Trans. Rehab. Eng., vol. 8, pp. 233-243, June 2000.

[22] J. Rasmussen, S. T. Christensen, M. Gföhler, M. Damsgaard, and T. Angeli, "Design optimization of a pedalling mechanism for paraplegics," Struct. Multidisc. Optim., vol. 26, pp. 132-138, 2004.

[23] E. S. Idsø, T. A. Johansen, and K. J. Hunt, "Finding the metabolically optimal stimulation pattern for FES-cycling," in Proc. 9th Ann. Conf. Int. Functional Electrical Stimulation Soc., (Bournemouth, England), pp. 239-241, 2004.

[24] M. Gföhler and P. Lugner, "Dynamic simulation of FES-cycling: influence of individual parameters," IEEE Trans. Neur. Sys. Rehab. Eng., vol. 12, pp. 398-405, December 2004.

[25] M. Gföhler, T. Angeli, T. Eberharter, P. Lugner, W. Mayr, and C. Hofer, "Test Bed with Force-Measuring Crank for Static and Dynamic Investigations on Cycling by Means of Functional Electrical Stimulation," IEEE Trans. Neur. Sys. Rehab. Eng., vol. 9, pp. 169179, June 2001.

[26] K. J. Hunt, B. Stone, N.-O. Negård, T. Schauer, M. H. Fraser, A. J. Cathcart, C. Ferrario, S. A. Ward, and S. Grant, "Control strategies for integration of electric motor assist and functional electrical stimulation in paraplegic cycling: utility for exercise testing and mobile cycling," IEEE Trans. Neural Sys. Rehab. Eng., vol. 12, pp. 89-101, March 2004.

[27] T. J. Barstow, A. M. E. Scremin, D. L. Mutton, C. F. Kunkel, T. G. Cagle, and B. J. Whipp, "Peak and kinetic cardiorespiratory responses during arm and leg exercise in patients with SCI," Spinal Cord, vol. 38, pp. 340-345, 2000. 